\title{
ECHINOCANDINS VERSUS DATED ANTIFUNGALS IN COMBINATION AGAINST OPPORTUNISTIC MYCOTIC INFECTIONS
}

\author{
MRRIDULA DANGI NARWAL, MEENAKSHI BALHARA, RENU CHAUDHARY, CHHILLAR AK*
}

\author{
Department of Biotechnology , Centre for Biotechnology, M.D. University, Rohtak, Haryana, India. Email: anil.chhillar@gmail.com
}

Received: 19 January 2017, Revised and Accepted: 03 February 2017

\begin{abstract}
Scientific and clinical reports globally demonstrated that the opportunistic mycotic infections are at major risk to the human fitness. In the past few decades, the development of resistance in microbes to existing antifungals has emphasized on the search of new antimycotic drugs. As a matter of fact, "echinocandins" are new categories of broad-spectrum antifungal enlighten a hope in this direction. Echinocandins are bulky lipopeptides that inhibit the production of $\beta$-[1,3]-glucan "a major constituent of fungal cell wall" which ultimately leads to the death of fungal pathogens. In vitro as well as in vivo published reports have demonstrated that the echinocandins exhibit fungicidal activity against most Candida spp., while fungistatic against Aspergillus spp. and exclusively found to be more effective when tested in combination with polyenes/azoles. The present article is expert views on the recent and historical literature available on the antifungal therapies with accessing their impact on the human health. Emphasis is given to the utility of the echinocandins a potental antifungal agent by discussing recent examples of clinical and laboratory studies including the use of improved proteomics approaches to know a bit more about the interaction of human host and fungal pathogens.
\end{abstract}

Keywords: Echinocandins, Antifungal, Aspergillus, Candida, Opportunistic, Combination.

(C) 2017 The Authors. Published by Innovare Academic Sciences Pvt Ltd. This is an open access article under the CC BY license (http://creativecommons. org/licenses/by/4. 0/) DOI: http://dx.doi.org/10.22159/ajpcr.2017.v10i8.17186

\section{INTRODUCTION}

A normal resident flora of the body also includes pathogenic microbes, which develop disease only when host immune defense is weak. Opportunistic mycoses are the type of fungal infections mostly occurring in the host with compromised immune system. Over the past few decades, the incidence of invasive fungal infections due to opportunistic pathogens has been escalated significantly [1]. This upsurge in infections is coupled with extreme morbidity/mortality [2], also the individuals undertaking blood/bone marrow transplantation, solid organ transplantation, and major surgery are at higher risk of such kind of infections [2,3]. Highest frequencies of opportunistic fungal infections are due to different species (spp.) of Candida (Candidiasis and Candidaemia) and Aspergillus (Aspergillosis). Current medical treatments are of limited utility, and their therapeutic use has been complicated by Candidaemia and invasive Candidiasis (IC), which contribute toward high morbidity and mortality [4]. Candidaemia is the fourth major type of microbial bloodstream infection [5], while Candida albicans being the major causative spp. for the development of IC [6]. Over the past two decades, the prevalence of Candidaemia has boosted as per the hospital records, which has shown the incidence ranging from $24 \%$ to $57 \%$. In a report out of 670 cases of Candidaemia, $274(41 \%)$ were hospital acquired, among which mortality turned out to be $39 \%$ [7] Both C. albicans and Candida non-albicans species are known to colonize the skin, gastrointestinal tract and reproductive tract in humans apart of Candidaemia, Invasive Aspergillosis (IA) which is caused by spp. of Aspergillus (Aspergillus fumigatus, Aspergillus flavus, Aspergillus terreus, and Aspergillus nidulans), has also been emerged as a major life risk in immunocompromised patients [8]. Recently, in an 18 month surveillance program, among 5.561 patients, only 12 patients were reported to be suffering from IA $(0.2 \%)$. However, mortality was found to be $60 \%$ [9]. Furthermore, researchers affirmed $7 \%$ of IA affected cases by a mortality rate of $91 \%$. Remarkably, $70 \%$ of these cases had been observed with no predisposing factors for opportunistic mycotic infections [10].

At present, major drugs used for systemic therapy of opportunistic fungal infections have different main targets: The polyenes and azoles affect the plasma membrane, whereas the antimetabolite, 5-fluorocytosine restricts DNA and RNA synthesis whereas echinocandins acts on the cell wall of the pathogens. Flucanozole targets lanosterol 14-alphademethylase and hinder the biosynthesis of ergosterol [11]. Since the first polyene was standardized 40 years earlier, the echinocandins were approved in January 2001 as a new class of antifungal drug. Different types of echinocandins include micafungin (MCF) (Mycamine, Astellas) which was accepted in 2005 and anidulafungin (ANF) (VER002, V-echinocandin, LY303366, Vicuron Pharmaceuticals) accepted in 2006; further, these two classes of echinocandin have been approved by the federal drug administration [12]. Since 2000, echinocandins have been extensively used for the cure and prophylaxis of invasive mycoses (IM) [13] and majorly suggested as first-line treatment for Candidiasis [14].

Echinocandins reveal effectiveantifungal activityagainstmajorinfectious fungi, such as Candida spp., Aspergillus spp., and Pneumocystis carinii. However, in case of Cryptococcus neoformans, current echinocandins lack in vitro activity. Although, semisynthetic echinocandins are on more beneficial sides like favorable pharmacokinetics allowing oncedaily administration low toxicity and fast antifungal activity. The echinocandins lately accessible for clinical purposes are caspofungin (CAS), MCF, and ANF [15]. Results of clinical practices applied in China suggest that CAS has been superior to the other two antifungal drugs classes: Polyenes and azoles, because of its efficiency in curing fungal infections (15\% superior to fluconazole); minor adverse effects such as hepatic dysfunction, infusion-related reaction and vomiting (25-50\% lower frequency); rapid resolution of symptoms (almost 3 days faster than Amphotericin B [AmpB]); and no antagonism in combination therapy [16].

Constant efforts are needed to develop the suboptimal remedial results linked with mycotic infections. However, monotherapy is often diagnosed with increased risk of toxicity, lesser resistance, or limited spectrum of activity. These limitations of monotherapy led to determine efficacy of combination therapy for the treatment and management of IM [17]. There are various probable benefits to combination antifungal therapy, i.e., wide spectrum and effectiveness of drug activity, speedy antifungal effect, synergy, limited dose, and chance of antifungal 
resistance is reduced. Although every antifungal agent has its own restrictions, therefore, combination therapy might become more efficient for the treatment of IM [18].

In this article, we have emphasized on echinocandins being potential source as an antifungal agent, their improved efficacy in combination with currently available antifungals including pharmacokinetics investigations have been discussed along with available reports on genomics and proteomics based studies, for elucidating the mechanism of action of these antifungal drugs.

\section{ECHINOCANDINS: THE POTENTIAL SOURCE AS AN ANTIFUNGAL AGENT}

Recently, only a few antifungals are in use that has significant efficacy against fungal infections. These antifungal drugs affect specifically the components constituting cell membrane of fungi or its biosynthetic pathways. However, more recent class of antifungals in use is echinocandins [19], which disrupts cell wall components by noncompetitively inhibiting the synthesis of $1,3-\beta$-glucans. First reported echinocandin was CAS, followed by MCF and ANF. CAS is found to be most effective against candidal esophagitis and candidemia, along with salvage therapy of Aspergillus infections and for empirical therapy of febrile neutropenia. Likewise, MCF is used in treating candidal esophagitis and cases of hematopoietic stem cell transplants. ANF has also been considered an effective drug to cure candidal esophagitis, Candidaemia [20].

Echinocandins seems the smart new choice for the cure of IM infections because of their reduced toxicity and slight drug-drug interactions. They are found to be fungicidal against yeast and fungistatic against mold. However, their price may reduce their usage during initial therapy in cases with fungemia in medical centers or ICU with a high rate of triazole-resistant Candida infections. Reportedly, Echinocandins act fungicidal against Candida spp. along with triazole-resistant isolates and show fungistatic activities against Aspergillus spp. echinocandins also reveal concentration-dependent activity against Candida spp. Although echinocandins are fungistatic against mold, according to one study, they might prove promising for treating opportunistic infections when administered in combination with $\mathrm{AmpB}$ or wide-spectrum triazoles, such as voriconazole. Reviews suggest that in clinical trials, CAS exhibit efficacy in curing eosophageal candidiasis, candidemia, and febrile neutropenia. However, MCF seems effective in hematopoietic stem cell transplant recipients and the treatment of esophageal candidiasis. ANF received approved labeling from the Food and Drug Administration in February 2006 [21].

Since 2000s, echinocandins are extensively recommended for the treatment of invasive fungal infections, remarkably, and IC. Although, cases of advance candidiasis in hosts, taking echinocandins, have been detected; however, the rare incidence of clinical failure is observed due to the possession of resistance by a generally susceptible Candida spp. isolate [13]. Reportedly, in vitro activity of antifungal drugs was tested against 496 isolates of yeasts and molds, during 2010-2012. Fks hot spots were sequenced for strains resistant to echinocandins and resistance was found infrequent among 8 Candida spp. and was only spotted in 3 isolates of Candida glabrata, having mutations in fks1 (F625S) and fks2 (S663P) [22].

Another study of 163 hosts during 2011-2012, revealed the fact that echinocandin failure was rare and molecular investigations of the fks1 and fks2 hotspots of the C. glabrata, discovered mutations only in 2 isolates (L628R and S629P in fks1) [23].

Furthermore, a report comprising data from 2008 to 2013 suggested that, among 1.380 hosts of C. glabrata, 3.1\% were resistant to ANF, $3.3 \%$ were non-responsive to CAS and $3.6 \%$ were not affected while treated with MCF [24].

\section{Mechanism of resistance}

Echinocandin resistance in C. glabrata is associated entirely with fks $1 \mathrm{p}$ and fks $2 \mathrm{p}$ amino acid substitutions [25]. Echinocandin failure accredited to mutations in fks1 is linked with raised chitin levels and the lack of a compensatory upsurge in chitin level on echinocandin introduction (Table 1) [26]

\section{MONOTHERAPY/COMBINATION THERAPY OF ECHINOCANDINS AND THEIR CHEMICALLY MODIFIED DERIVATIVES}

\section{Echinocandins in combination with polyenes}

Recently, combination antifungal therapy of echinocandins with polyenes has been explored in nonclinical studies for boosting the effect of the treatment for IA. AmpB aims at ergosterol present in fungal cell membrane by binding with it consequently creating pores in the membrane and disrupting membrane integrity, resulting in fungal cell lysis [27]. However, the echinocandins mainly focus the enzyme 1,3- $\beta$-Dglucan (BG) synthetase, required for the synthesis of $1,3-\mathrm{BG}$, a vital component of fungal cell wall [28]. The specificity of the echinocandins against the fungal cell wall also seems promising for least hazardous side effects. Hence, combination of a drug, which aims at the cell wall and the one, targeting the plasma membrane, might result in additive or synergistic antifungal effects. Nonclinical reports suggest that the combination of AmpB and the echinocandin does not act antagonistic during the treatment of Aspergillus infections [29].

Indeed, enhanced efficiency has been displayed by mice model (affected with chronic granulomatous disease and pulmonary IA) when administrated with combination of MCF (Mycamine) and AmpB [30] and in case of murine systemic aspergillosis, increased efficacy has been reported when treated with a combination of CAS plus AmpB or intra-lipid AmpB (LAmpB) [31]. Whereas, Clemons and Stevens [29] reported that there was no synergistic activity of MCF and AmpB for pulmonary IA in immunocompromised DBA/2 mice, although there was no antagonism too.

In another case of a Systemic Murine Aspergillosis model [32], the researchers observed narrow additive effects of suboptimal doses of LAmpB and MCF with appreciably decreased fungal load in the spleens when treated with LAmpB before MCF. Although for the disseminated and pulmonary murine infections, administering LAmpB along with MCF or CAS, was reported to be neither antagonistic or additive nor synergistic although improved survival or decrease in fungal load also considered. However, Wasan et al. [33] observed additive effects in a rat model (infected with $A$. fumigatus) when it was exposed LAmpB $(5 \mathrm{mg} / \mathrm{kg}$ ) plus CAS (3 mg/kg), parallely. In this study, the researchers reported that the combination appreciably reduced colony forming unit (CFU) (by 98\%), while LAmpB or CAS monotherapy reduced $69 \%$ and $80 \% \mathrm{CFU}$, respectively, in comparison with untreated controls.

Instead of combination therapy, researchers analyzed the decline of fungal load in kidneys during disseminated infection, was appreciably improved when LAmpB was administered previous to CAS instead of CAS was given first (sequential therapy) [34].

In conclusion, treatment of disseminated or pulmonary infection of A. fumigatus in mice, with LAmpB + echinocandin or exposure of LAmpB previous to the echinocandins, was equally efficient as LAmpB treatment alone, but with prolonged survival and reduced fungal load in the target tissues. In contrast, unlike pulmonary infection, in the hosts of disseminated infection, survival was prolonged by echinocandin monotherapy, and in general, did not considerably decrease the fungal load compared to LAmpB. Taken both models into account, there was appreciably improved effect when LAmpB was given earlier to the echinocandins; these explanations deliver a convincing evidence for using LAmpB first if sequential therapy is used for IA [35].

\section{Echinocandins in combination with azoles}

A research, including 101 cases (79 proven, 22 probable), across four risk groups (patients with hematologic malignancy, stem cell recipients, 
Table 1: Mechanism of resistance of pathogenic species against diverse antifungal drugs [12]

\begin{tabular}{|c|c|c|c|c|}
\hline Class & Drugs & Targets & Resistant species & Mechanism of resistance \\
\hline Polyenes & $\begin{array}{l}\text { Amphotericin B } \\
\text { Nystatin } \\
\text { Natamycin }\end{array}$ & $\begin{array}{l}\text { Binds with ergosterol } \\
\text { present in plasma } \\
\text { membrane }\end{array}$ & $\begin{array}{l}\text { Candida lusitaniae, Candida } \\
\text { lipolytica, Candida guillermondii, } \\
\text { Trichosporon beigelii, } \\
\text { Pseudallescheria boydii and } \\
\text { Scopulariopsis }\end{array}$ & $\begin{array}{l}\text { Decrease in the ergosterol content due to the } \\
\text { absence of the enzyme, D-isomerase } \\
\text { Alteration in } \beta-1,3 \text { glucans }\end{array}$ \\
\hline Azoles & $\begin{array}{l}\text { Ketaconazole } \\
\text { Fluconazole } \\
\text { Itracoazole } \\
\text { Voriconazole } \\
\text { Miconazole } \\
\text { Posaconazole } \\
\text { Ravuconazole }\end{array}$ & $\begin{array}{l}\text { Binds with lanosterol } \\
\text { demethylase in plasma } \\
\text { membrane }\end{array}$ & $\begin{array}{l}\text { Candida neoformans, Candida } \\
\text { albicans, Candida dubliniensis, } \\
\text { Candida norvegensis, and Candida } \\
\text { inconspicua }\end{array}$ & $\begin{array}{l}\text { Alteration or overexpression of the target } \\
14-\alpha \text {-DM enzyme } \\
\text { Mutation in the ergosterol } \\
\text { pathway [defective } \alpha-5,6 \text {-desaturase, encoded } \\
\text { by the gene ERG3] } \\
\text { Amino acid substitutions }\end{array}$ \\
\hline Antimetabolite & Flucytosine & $\begin{array}{l}\text { Act as scavenger for } \\
\text { salvage pathway }\end{array}$ & $\begin{array}{l}\text { Candida spp. Candida neoformans, } \\
\text { Candida albicans }\end{array}$ & $\begin{array}{l}\text { Mutational decline of cytosine permease or } \\
\text { deaminase activity } \\
\text { Loss of activity of enzyme [uracil } \\
\text { phosphoribosyl transferase] }\end{array}$ \\
\hline Echinocandins & $\begin{array}{l}\text { Caspofungin } \\
\text { Micafungin } \\
\text { Anidulafungin }\end{array}$ & $\begin{array}{l}\text { Non-competitive } \\
\text { inhibition of cell wall } \\
\text { protein } \beta \text {-1,3-glucan } \\
\text { synthase }\end{array}$ & Candida glabrata & $\begin{array}{l}\text { Fks1p and fks2p amino acid substitutions } \\
\text { Deletion of genes coding for components } \\
\text { related with cell wall integrity, such as WSC1, } \\
\text { PKC1, BCK1, and SLT2 }\end{array}$ \\
\hline
\end{tabular}

solid organ transplantation hosts and other), and two periods, P1 (1995-2003) and P2, (2004-2011); revealed that amid periods, the fraction of patients, diagnosed with hematologic malignancy or solid organ transplant recipients, intensity from $47 \%$ to $73 \%$, while "other" declined from $33 \%$ to $11 \%$ with probability (p) of 0.036 . However, between periods, the cumulative prevalence of invasive infections did not appreciably escalated in stem cell transplant recipients $(\mathrm{p}=0.27)$ or solid organ transplant recipients $(\mathrm{p}=0.30)$. Notably, more patients were given amphotericin-echinocandin combination therapy in P2 (31\% vs. $5 \%, \mathrm{p}=0.01$ ); though, no improvement was observed during 90 -days survival $(54 \%$ vs. $59 \%, \mathrm{p}=0.67)$ [34].

Combination therapy of pediatric IM has hardly been reported. In a research, conducted on 19 children (with a median age of 5.3) affected with IM, were administered with liposomal LAmpB monotherapy for a median duration of 12 days (range 3-69 days). As patients were obstinate to LAmpB; therefore, CAS was supplemented in 11 patients. In the remaining 6 cases, LAmpB was stopped and a combination of CAS and voriconazole were commenced. 12-week survival rate of these patients was $75 \%$ with no hazardous side effect. Data suggest that combination antifungal therapy is safe and effective in children with hematological malignancies [36].

In a more recent study involving 10 randomized controlled trials, involving 2,837 hosts, echinocandins and triazoles exhibited parallel outcomes in terms of promising treatment success rate such that the relative risk (RR)found to be 1.02, fungal success rate with RR of 0.98 , advance infection RR to be 1.09 , drug-associated adversity with RR 0.94, and all-cause mortality ( $R R=0.85 ; 95 \%$ cumulative prevalence, $0.66-1.10)$. Moreover, echinocandins were found to be more efficient than triazoles in case of patients diagnosed with hematologic malignancies or hematopoietic stem cell transplantation recipients with RR of 1.08. Echinocandins appreciably reduced the adverse effects linked to the withdrawal rate in comparison with triazoles $(\mathrm{RR}=0.47)$ [37].

In a randomized, double-blind, placebo-controlled, multicenter trial from 93 international sites, 454 patients with hematologic malignancies or hematopoietic stem cell transplantation and proven or probable IA, were randomly given voriconazole and ANF. For combination therapy, mortality rates observed at 6 weeks, was $19.3 \%$ (26 of 135) and for voriconazole monotherapy, it was, $27.5 \%$ (39 of 142) ( $\mathrm{p}=0.087$ ); concluding that as compared to single drug administration, combination therapy of voriconazole with ANF exhibited greater survival in patients with IA [38].
In another research, it was found that combination therapy (MCF and daily dose of oral $400 \mathrm{mg}$ itraconazole) can be promising alternative for treatment of fatal IA of the sinus [39].

A study conducted in Germany from 2006 to 2012 in patients with acute alternative lymphoblastoma leukemia and hematopoietic stem cell transplantation recepients having IM. Out of 25 patients of acute lymphoblastoma leukemia along with 28 cases of suspected IM, 20 were administered with empirical CAS first line monotherapy (71.4\%), 5 were given second line monotherapy $(17.9 \%)$ and combination therapy in 3 cases $(10.7 \%)$ concluding that empirical CAS seems to be a successful therapeutic alternative in case of IM having acute lymphoblastoma leukemia with probable IM [40].

In an observational study conducted in Japanese patients, from July 2007 to June 2010, it was observed that among 241 patients, 86 patients (35.7\%), were diagnosed with 143 adverse drug reactions, especially hepatobiliary disorders. The clinical success rate was $72.8 \%$ (131/180 patients), and the prevalence of advance infections was merely 4.4\% (8/180 patients) concluding that MCF had satisfactory efficiency against IM in Japanese episodes undergoing hematopoietic stem cell transplantation [41].

Present recommendations acclaim antifungal measures for children who are at high risk for IM, though the administration of polyenes and triazoles may not be recommended in some episodes due to toxicities and drug-drug interactions. Although Azole antimycotic drugs are administered clinically in patients with IM, these agents have been detected with renal toxicity thus causing vomiting. Thus it has led to requirement of new antifungal agents with minimum toxic effects, although an investigation for the noval antimycotic metabolites remains new area of research [42]. In an analysis, including 21 IM affected children with a median age of 9 , it was concluded that receiving MCF between 3 and $4 \mathrm{mg} / \mathrm{kg}$ of body weight, two times every week, could be an appropriate, nontoxic and effective substitute for antifungal prophylaxis [43].

Recently, Antonio Roseto and coworkers reported that ANF exhibited a convincing in vitro activity against both planktonic and biofilms cells. This analysis involved nine Candida strain biofilms: Four C. albicans, two C. glabrata and three Candida guilliermondii and it was found that ANF alone was active against biofilms whereas ANF in combination with three non-steroid anti-inflammatory drugs (NSAIDs): Aspirin, diclofenac and ibuprofen, proved synergistic in action against Candida 
spp. biofilms. These findings may suggest new therapeutic stratergies of ANF with NSAIDs against Candida biofilm-related IM [44].

\section{Echinocandin in combination with adjunctive agents}

Hematopoietic growth factors encourage the proliferation and stimulation of phagocytic host cells; out of these, colony-stimulating factor (G-CSF), GM-CSF, and M-CSF have been considered as adjunctive immunomodulatory agents against fungal pathogens.

G-CSF incites the production and differentiation of myeloid progenitor cells to polymorph nuclear leukocytes, such that raising the quantity of mature neutrophils. Moreover, In vitro G-CSF boosts the phagocytic activity of neutrophils along with respiratory burst against Aspergillus spp. [44,45] In neutropenic animal models of IA, G-CSF treatment was coupled with improved survival as well as quicker recovery from neutropenia [46].

\section{ADVANCE THERAPIES FOR BETTER TREATMENT STRATEGIES AND NEED FOR NOVEL DRUG TARGET IDENTIFICATION}

As most of the pathogenic spp. of Candida and Aspergillus has developed resistance against standard antifungal drugs, there is a need to search advance therapies for better treatment of IM. Recently, a novel echinocandin-type antifungal metabolite, MIG0310, with a molecular formula $\mathrm{C}(48) \mathrm{H}(66) \mathrm{O}(18)$ was isolated and characterized, obtained originally from fungal strain, Fusarium MS-R1, has led to the idea of developing new antifungal drugs against resistant Candida spp. [47].

Another interesting research showed that an inhibitor of Hos2 fungal histone deacetylase named as MGCD290 employs a noticeably satisfactory impact on the minimum inhibitory concentrations (MICs) of fluconazole and the echinocandins, showing synergy when administered in combination, converting resistant Candida spp. to susceptible, irrespective of fks mutations [48].

In 2015, Wiederhold et al. [49] confirmed in vitro and in vivo antifungal activity of arylamidine, T-2307 against $C$. albicans, resistant to echinocandin along with usefulness of the untried echinocandin ASP9726 in a guinea pig affected with IA. Guinea pigs were subcutaneously injected with investigational echinocandin at different concentrations, and plasma concentrations were noted. Immunocompromised guinea pigs were infected with $A$. fumigatus and three drugs (ASP9726, voriconazole, CAS) were given for 8 days. Measurement of variations in fungal burden showed that ASP9726 plasma concentrations were raised proportionally with every dose, and the drug was well tolerated at every dosage. Every dosage of ASP9726, voriconazole, and CAS appreciably reduced the infection. Similarly, ASP9726 at $5 \mathrm{mg} / \mathrm{kg}$ had considerably improved survival [50].

Current epidemiological investigations have disclosed rise in number of C. glabrata revealing reduced echinocandin sensitivities. The significant contribution in maintaining integrity of fungal cell wall is done by the Slt2 mitogen-activated protein kinase pathway. Some transcription factors such as Rlm1 and Swi4-Swi6 cell cycle box binding factor (SBF) have been reported downstream of Slt2 such that Slt2 and Rlm1 display significant response to MCF exposure, though, no such data available for SBF in C. glabrata. Hence, researchers produced some C. glabrata strains with the lack of SBF ( $\Delta$ swi4 and $\Delta$ swi6 strains) or overexpressing SBF (SWI4 and SWI6 strains) and estimated their susceptibilities against MCF. MCF resistance substantially declined in the $\Delta$ swi4 strain, whereas it amplified in SWI4 strains concluding that although Swi4 is principally responsible for MCF resistance more than Swi6 in C. glabrata. Moreover, the overexpression of RLM1 stimulated added MCF tolerance in the wild-type strains, but not in the $\Delta$ swi4 and $\Delta$ swi6 strains, proposing that Rlm 1 and SBF act collectively in response to MCF administration [51].

In a study conducted on 257 patients, with probable IC and getting ANF administration, were evaluated clinically and microbiologically along with measuring their serum $(1,3)$-BG serially. BG can be used as a biomarker for IC. Correlation of initial and final BG levels with overall outcome was assessed in each patient and success rate found to be $85 \%$. Success of treatment was correlated with a reduction in BG levels and early BG of $<416 \mathrm{pg} / \mathrm{ml}$ can predict promising treatment result especially for patients with IC receiving predominantly echinocandin therapy [52].

In another study, BG surveillance in ICU patients infected with IC getting ANF administration showed that ICU patients with proven or probable IC have higher BG levels than ICU patients without IM. Preemptive ANF was nontoxic and often successfully tolerated with exceptional results. BG surveillance may be effectul in diagnosing ICU patients with a greater probability of developing IM [53].

\section{LATEST TECHNIQUES FOR MULTIFACTOR ANALYSIS INCLUDING PROTEOMIC DATA}

Non-ribosomal peptide synthetases (NRPSs) are the main enzymes in fungal peptidic association and are accountable for some of the best-known, supreme beneficial and record devastating fungal metabolites. Most of NRPS encoding genes of any fungal spp. can now be identified by genome sequencing and recognizing the catalytic domains using bioinformatics. Genomic sequencing and annotation has lately categorized the gene clusters accountable for two significant classes of NRP fungal derivative drugs, cyclosporine and the echinocandins. Genetic mapping of these gene assemblies has been done and characterization of their function is still in progress. Genomic sequencing of wild type and variants of echinocandin family has made it possible to understand the fundamental architecture of echinocandin NRPS pathways [54].

In a study comparing the echinocandins activity against 7 C. albicans, 5 C. dubliniensis, and 2 C. africana spp. by time-kill analysis, MIC values were found to be similar for the $3 \mathrm{spp}$. as echinocandins displayed weak killing activity and no mortality against $C$. africana. This study suggests time - kill analysis can be taken as a promising diagnostic tool for identification of new pathogenic spp. especially when echinocandin therapy collapses [55].

Recently, liquid chromatography-tandem mass spectrometry assay has been used for determining plasma level of MCF, administered in patients affected with IC. Assay timings were 20 minutes while the quantification was done at the lower limit of $0.1 \mathrm{mg} / \mathrm{ml}$. This assay provides high specificity, accuracy and precision, can be a promising technique for quantification of plasma level of MCF [56].

However, a gel-free technique of isobaric tagging for relative and absolute quantitation [iTRAQ] is now accessible to get greater quantities of recognized proteins and to provide in-detail analysis. This technique practices a sequence of isobaric tags which links with lysines and the $\mathrm{N}$ terminal of every protein succeeding proteolysis $[57,58]$. Recently, the proteomic analysis of $A$. fumigatus against CAS has been estimated by ITRAQ technique to determine possible biomarkers of drug efficacy [59]. Using iTRAQ out of 471 identified proteins 122 proteins displayed at least a 2 -fold difference in relative richness after exposure to CAS at just below the minimum effective concentration [0.12 $\mathrm{g} / \mathrm{ml}]$ [60]. Lately, the major fluctuations have been observed in a protein [AFUA_1G12250] in mitochondria, whose intensity reduced more than 16 times in the secreted portion. Earlier studies revealed the probable importance of this protein as a biomarker exclusively to CAS as it was not reported while A. fumigatus was exposed to either voriconazole or AmpB [61-62].

Another is ChiA1, whose level declined 12.1 times in the cell wall/membrane portion. Previously, the protein encoded by Pma1 gene was reported to be raised 2 times against AmpB while reduced 4.3 times and 5.1 times after CAS exposure as determined by proteomics and microarray analysis [61]. The level of the key allergen and cytotoxin AspF1 has also been observed to drop by 12.1-fold after the supplement of the drug. A successive iTRAQ evaluation of an echinocandin-resistant 
strain [fks1-S678P] was taken to authenticate proteins unambiguous to drug action. Also, Microarray analysis of non-resistant strain has been accomplished for assessment of the link that associates proteomics with genomics such that overall 117 genes were discovered which vary minimum by 2 times. However, 22 proteins with noteworthy deviations were acknowledged by iTRAQ also displayed substantial alterations in their level of gene expression analysed by microarray [61].

Recent studies used the combination of matrix-assisted laser desorption ionization-time of flight mass spectroscopy and two-dimensional polyacrylamide gel electrophoresis to recognize the modifications in protein richness in a strain of C. albicans following treatment with CAS. Mainly the proteins involved in glycolysis and gluconeogenesis gave response to CAS exposure, while other proteins reported to respond specifically to this drug found to be involved in cell stress and heat shock, and the proteins of the Kreb's cycle and amino acid biosynthesis. Some enzymes catalyzing cell wall biosynthesis and the regulating BG synthase were also acknowledged [62].

\section{FUTURE PROSPECTIVE}

The medical practice with echinocandin drugs has been highly successful, as this class of drugs exhibit strong efficacy, especially with Candida spp., with negligible side effects and a low frequency of resistance. In future, its effect in combination with chelators can be explored. Moreover, it might give significant results with adjunctive immunotherapic agents under which research is under dogged. Echinocandins can be promising for novel drug design and might prove a milestone for the pharmaceutical industry in future.

\section{ACKNOWLEDGMENT}

The authors would like to thank Maharishi Dayanand University for necessary support.

\section{REFERENCES}

1. Frange P, Bougnoux ME, Lanternier F, Neven B, Moshous D, Angebault $\mathrm{C}$, et al. An update on pediatric invasive aspergillosis. Med Mal Infect 2015;45(6):189-98.

2. Taccone FS, Van den Abeele AM, Bulpa P, Misset B, Meersseman W, Cardoso T, et al. Epidemiology of invasive aspergillosis in critically ill patients: Clinical presentation, underlying conditions, and outcomes. Crit Care 2015;19:7.

3. Curbelo J, Galván JM, Aspa J. Updates on Aspergillus, Pneumocystis and other opportunistic pulmonary mycoses. Arch Bronconeumol 2015;51:647-53

4. Neoh CF, Slavin M, Chen SC, Stewart K, Kong DC. Echinocandins in the treatment of candidaemia and invasive candidiasis: Clinical and economic perspectives. Int J Antimicrob Agents 2014:43:207-14.

5. Lackner M, Tscherner M, Schaller M, Kuchler K, Mair C, Sartori B, et al. Positions and numbers of FKS mutations in Candida albicans selectively influence in vitro and in vivo susceptibilities to echinocandin treatment. Antimicrob Agents Chemother 2014;58(7):3626-35.

6. Lum KY, Tay ST, Le CF, Lee VS, Sabri NH, Velayuthan RD, et al. Activity of novel synthetic peptides against Candida albicans. Sci Rep 2015;5:9657.

7. De Rosa FG, Corcione S, Filippini C, Raviolo S, Fossati L, Montrucchio $\mathrm{C}$, et al. The effect on mortality of fluconazole or echinocandins treatment in candidemia in internal medicine wards. PLoS One 2015;10(5):e0125149.

8. Thakur R, Anand R, Tiwari S, Singh AP, Tiwary BN, Shankar J. Cytokines induce effector T-helper cells during invasive aspergillosis; What we have learned about T-helper cells? Front Microbiol 2015;6:429.

9. Montagna MT, Caggiano G, Lovero G, De Giglio O, Coretti C, Cuna T, et al. Epidemiology of invasive fungal infections in the intensive care unit: Results of a multicenter Italian survey (AURORA project). Infection 2013;41(3):645-53.

10. Meersseman W, Vandecasteele SJ, Wilmer A, Verbeken E, Peetermans WE, Van Wijngaerden E. Invasive aspergillosis in critically ill patients without malignancy. Am J Respir Crit Care Med 2004;170(6):621-5.

11. Medeiros CI, Silva DD, Filho GG, Filho AA, Lima EE. Activity anti-c. Tropicalis and effects of the combination of (s)-(-)-citronellal with four antifungal applied in vulvovaginal candidiasis. Int J Pharm Pharm Sci 2016;8(8):347-51

12. Ruhil S, Balhara M, Dhankhar S, Kumar V, Chhillar AK. Invasive aspergillosis: Adjunctive combination therapy. Mini Rev Med Chem 2012;12(12):1261-72.

13. Fekkar A, Dannaoui E, Meyer I, Imbert S, Brossas JY, Uzunov M, et al. Emergence of echinocandin-resistant Candida spp. In a hospital setting: A consequence of 10 years of increasing use of antifungal therapy? Eur J Clin Microbiol Infect Dis 2014;33(9):1489-96.

14. González de Molina F, Martínez-Alberici Mde L, Ferrer R. Treatment with echinocandins during continuous renal replacement therapy. Crit Care 2014;18(2):218.

15. Stan CD, Tuchilus C, Stan CI. Echinocandins - new antifungal agents. Rev Med Chir Soc Med Nat Iasi 2014;118(2):528-36.

16. Zhang C, Cheng J, Jiang Y, Liu J. Application of caspofungin in China compared with amphotericin B and fluconazole. Ther Clin Risk Manag 2014;10:737-41.

17. Bolard J. How do the polyene macrolide antibiotics affect the cellular membrane properties? Biochim Biophys Acta 1986;864(3-4):257-304.

18. Steinbach WJ, Juvvadi PR, Fortwendel JR, Rogg LE. Newer combination antifungal therapies for invasive aspergillosis. Med Mycol 2011;49 Suppl 1:S77-81.

19. Prasad R, Shah AH, Rawal MK. Antifungals: Mechanism of action and drug resistance. In: Jose R, Hana S, Maik K, editors. Yeast Membrane Transport. Vol. 892. Switzerland: Springer; 2016. p. 327-49.

20. Morrison VA. Echinocandin antifungals: Review and update. Expert Rev Anti Infect Ther 2006;4(2):325-42.

21. Morris MI, Villmann M. Echinocandins in the management of invasive fungal infections, Part 2. Am J Health Syst Pharm 2006;63(19):1813-20.

22. Pfaller MA, Messer SA, Jones RN, Castabheira M. Antifungal susceptibilities of Candida, Cryptococcus neoformans and Aspergillus fumigatus from the Asia and Western Pacific region: Data from the SENTRY antifungal surveillance program (2010-2012). J Antibiot 2015;68 Suppl 9:556-61.

23. Matsumoto E, Boyken L, Tendolkar S, McDanel J, Castanheira M, Pfaller M, et al. Candidemia surveillance in Iowa: Emergence of echinocandin resistance. Diagn Microbiol Infect Dis 2014;43 Suppl 3:207-14.

24. Pham CD, Iqbal N, Bolden CB, Kuykendall RJ, Harrison LH, Farley MM. Role of FKS mutations in Candida glabrata: MIC values, echinocandin resistance, and multidrug resistance. Antimicrob Agents Chemother 2014;44 Supp1 2:96-104.

25. Dudiuk C, Gamarra S, Leonardeli F, Jimenez-Ortigosa C, Vitale RG, Afeltra J, et al. Set of classical PCRs for detection of mutations in Candida glabrata FKS genes linked with echinocandin resistance. J Clin Microbiol 2014;52:2609-14.

26. Walker LA, Gow NA, Munro CA. Elevated chitin content reduces the susceptibility of Candida species to caspofungin. Antimicrob Agents Chemother 2013;57(1):146-54.

27. Czub J, Baginski M. Modulation of amphotericin B membrane interaction by cholesterol and ergosterol - A molecular dynamics study. J Phys Chem B 2006;110:16743-53.

28. Stone EA, Fung HB, Kirschenbaum HL. Caspofungin: An echinocandin antifungal agent. Clin Ther 2002;24(33):351-77.

29. Clemons KV, Stevens DA. Efficacy of micafungin alone or in combination against experimental pulmonary aspergillosis. Med Mycol 2006;44:69-73.

30. Dennis CG, Greco WR, Brun Y, Youn R, Slocum HK, Bernacki RJ. Effect of amphotericin B and micafungin combination on survival, histopathology, and fungal burden in experimental aspergillosis in the p47phox/mouse model of chronic granulomatous disease. Antimicrob Agents Chemother 2006;50:422-7.

31. Adler-Moore JP, Proffitt RT. Amphotericin B lipid preparations: What are the differences? Clin Microbiol Infect 2008;14 Suppl 4:25-36.

32. Graybill JR, Bocanegra R, Gonzalez GM, Najvar LK. Combination antifungal therapy of murine aspergillosis: Liposomal amphotericin B and micafungin. J Antimicrob Chemother 2003;52:656-62.

33. Wasan KM, Sivak O, Rosland M, Risovic V, Bartlett K. Assessing the antifungal activity, pharmacokinetics, and tissue distribution of amphotericin B following the administration of Abelcet and AmBisome in combination with caspofungin to rats infected with Aspergillus fumigatus. J Pharm Sci 2007;96(7):1737-47.

34. Abidi MZ, Sohail MR, Cummins N, Wilhelm M, Wengenack N, Brumble L, et al. Stability in the cumulative incidence, severity and mortality of 101 cases of invasive mucormycosis in high-risk patients from 1995 to 2011: A comparison of eras immediately before and after the availability of voriconazole and echinocandin-amphotericin 
combination therapies. Mycoses 2014;57(11):687-98.

35. Olson JA, George A, Constable D, Smith P, Proffitt R, Moore JP. Liposomal amphotericin B and echinocandins as monotherapy or sequential or concomitant therapy in murine disseminated and pulmonary Aspergillus fumigatus infections. Antimicrob Agents Chemother 2010;54:3884-94.

36. Yilmaz D, Balkan C, Ay Y, Akin M, Karapinar B, Kavakli K. A rescue therapy with a combination of caspofungin and liposomal amphotericin $\mathrm{B}$ or voriconazole in children with haematological malignancy and refractory invasive fungal infections. Mycoses 2011;54(3):234-42.

37. Wang JF, Xue Y, Zhu XB, Fan H. Efficacy and safety of echinocandins versus triazoles for the prophylaxis and treatment of fungal infections: A meta-analysis of RCTs. Eur J Clin Microbiol Infect Dis 2015;34(4):651-9.

38. Marr KA, Schlamm HT, Herbrecht R, Rottinghaus ST, Bow EJ, Cornely OA, et al. Combination antifungal therapy for invasive aspergillosis: A randomized trial. Ann Intern Med 2015;162:81-9.

39. Ogawa T, Matsumoto K, Tsujimoto K, Hishiya N, Yamada Y, Uno K, et al. Chronic invasive sinus and intracerebral aspergillosis controlled by combination therapy with micafungin and a daily dose of $400 \mathrm{mg}$ itraconazole oral solution. J Infect Chemother 2015;21(2):134-7.

40. Kiehl MG, Egerer G, Engelhardt M, Gross B. Empirical caspofungin therapy in clinical practice for suspected invasive fungal disease in adults with acute lymphoblastic leukaemia. Mycoses 2015;58(2):76-81.

41. Kobayashi C, Hanadate T, Niwa T, Hirano Y, Yoshiyasu T, So M, et al. Safety and efficacy of micafungin for prophylaxis against invasive fungal infections in Japanese patients undergoing hematopoietic stem cell transplantation: Results of a post-marketing surveillance study. J Infect Chemother 2015;21(6):438-43.

42. Nandhini SU, Bharathy PJ, Rekha S. Antifungal compounds from marine Streptomyces. Int J Pharm Pharm Sci 2015;7(1):207-9.

43. Bochennek K, Balan A, Müller-Scholden L, Becker M, Farowski F, Müller C, et al. Micafungin twice weekly as antifungal prophylaxis in paediatric patients at high risk for invasive fungal disease. J Antimicrob Chemother 2015;70(5):1527-30

44. Rosato A, Catalano A, Carocci A, Carrieri A, Carone A, Caggiano G, et al. In vitro interactions between anidulafungin and nonsteroidal antiinflammatory drugs on biofilms of Candida spp. Bioorg Med Chem 2016;24(5):1002-5.

45. Roilides E, Uhlig K, Venzon D, Pizzo PA, Walsh TJ. Enhancement of oxidative response and damage caused by human neutrophils to Aspergillus fumigatus hyphae by granulocyte colony-stimulating factor and gamma interferon. Infect Immun 1993;61(4):1185-93

46. Pursell K, Verral S, Daraiesh F, Shrestha N, Skariah A, Hasan E, et al. Impaired phagocyte respiratory burst responses to opportunistic fungal pathogens in transplant recipients: In vitro effect of r-metHuG-CSF (filgrastim). Transpl Infect Dis 2003;5:29-37.

47. Masaphy S. A novel echinocandin MIG0310 with anticandida activity from newly isolated Fusarium sp. Strain MS-R1. J Appl Microbiol 2014:116(6):1458-64.

48. Pfaller MA, Rhomberg PR, Messer SA, Castanheira M. In vitro activity of a Hos2 deacetylase inhibitor, MGCD290, in combination with echinocandins against echinocandin-resistant Candida species. Diagn Microbiol Infect Dis 2015;81(4):259-63.

49. Wiederhold NP, Najvar LK, Fothergill AW, Bocanegra R, Olivo M, McCarthy DI, et al. The novel arylamidine T-2307 maintains in vitro and in vivo activity against echinocandin-resistant Candida albicans. Antimicrob Agents Chemother 2015;59(2):1341-3.

50. Wiederhold NP, Najvar LK, Matsumoto S, Bocanegra RA, Herrera ML, Wickes BL, et al. Efficacy of the investigational echinocandin ASP9726 in a guinea pig model of invasive pulmonary aspergillosis. Antimicrob Agents Chemother 2015;59(2):2875-81.

51. Nagayoshi Y, Miyazaki T, Minematsu A, Yamauchi S, Takazono T, Nakamura S, et al. Contribution of the Slt2-regulated transcription factors to echinocandin tolerance in Candida glabrata. FEMS Yeast Res 2014;14(7):1128-31.

52. Jaijakul S, Vazquez JA, Swanson RN, Ostrosky-Zeichner L. (1,3)-ß-Dglucan as a prognostic marker of treatment response in invasive candidiasis. Clin Infect Dis 2012;55(8):521-6.

53. Hanson KE, Pfeiffer CD, Lease ED, Balch AH, Zaas AK, Perfect JR, et al. B-D-glucan surveillance with preemptive anidulafungin for invasive candidiasis in intensive care unit patients: A randomized pilot study. PLoS One 2012;7(4):e42282.

54. Bills G, Li Y, Chen L, Yue Q, Niu XM, An Z. New insights into the echinocandins and other fungal non-ribosomal peptides and peptaibiotics. Nat Prod Rep 2014;31(10):1348-75.

55. Gil-Alonso S, Jauregizar N, Cantón E, Eraso E, Quindós G. Comparison of the in vitro activity of echinocandins against Candida albicans, Candida dubliniensis, and Candida africana by time-kill curves. Diagn Microbiol Infect Dis 2015;82(1):57-61.

56. Cangemi G, Barco S, Bandettini R, Castagnola E. Quantification of micafungin in human plasma by liquid chromatography-tandem mass spectrometry. Anal Bioanal Chem 2014;406(6):1795-8.

57. Ross PL, Huang YN, Marchese JN, Williamson B, Parker K, Hattan S, et al. Multiplexed protein quantitation in Saccharomyces cerevisiae using amine-reactive isobaric tagging reagents. Mol Cell Proteomics 2004;3(12):1154-69.

58. Zieske LR. A perspective on the use of iTRAQ reagent technology for protein complex and profiling studies. J Exp Bot 2006;57(9):1501-8.

59. Cagas SE, Jain MR, Li H, Perlin DS. Profiling the Aspergillus fumigatus proteome in response to caspofungin. Antimicrob Agents Chemother 2011;55(1):146-54.

60. da Silva Ferreira ME, Malavazi I, Savoldi M, Brakhage AA, Goldman MH, Kim HS, et al. Transcriptome analysis of Aspergillus fumigatus exposed to voriconazole. Curr Genet 2006;50(1):32-44.

61. Gautam P, Shankar J, Madan T, Sirdeshmukh R, Sundaram CS, Gade WN, et al. Proteomic and transcriptomic analysis of Aspergillus fumigatus on exposure to amphotericin B. Antimicrob Agents Chemother 2008;52(12):4220-7.

62. Hoehamer CF, Cummings ED, Hilliard GM, Rogers PD. Changes in the proteome of Candida albicans in response to azole, polyene, and echinocandin antifungal agents. Antimicrob Agents Chemother 2010;54(5):1655-64. 